BY

CHARLES W. BUCKLEY, M.D., F.R.C.P.

PHYSICIAN TO THE DEVONSHIRE HOSPITAL FOR RHEUMATIC DISEASES, BUXTON: MEMBER OF THE BRITISH MEDICAL ASSOCIATION ARTHRITIS COMMITTEE

In the report of the B.M.A. Arthritis Committee a classification was adopted differentiating on broad lines rheumatoid arthritis and osteo-arthritis. This differentiation is of the utmost importance from.the point of view of aetiology, prognosis, and treatment, and as the subject is so wide I shall limit my remarks to the treatment of rheumatoid arthritis alone. This has been subdivided into primary and secondary; in the former no focus of infection can be found; in the latter such a focus can be identified and should be dealt with as radically as possible, with due regard to the general condition of the patient. It is essential to remember that wholesale extraction of teeth or enucleation of tonsils may be accompanied by as much shock as a major operation, and resistance may be so much lowered by such a procedure that the disease gains a firm hold, and the patient's condition is worse than before. This disastrous result may be, in some measure, caused by the liberation of toxins and micro-organisms in great amount, and their entrance into the general circulation. I shall not attempt to enumerate the many possible foci from which infection may proceed, but would stress the fact that the existence of a focus neecs to be associated with other factors before infection spreads to the joints or other tissues. The soil is, in fact, as important as the seed. Heredity is a factor in about 50 per cent. of cases, and the bodily characteristics are a slight, spare physique, the patient often being emotional and lacking in endurance, with poor circulation, cold extremities, and a liability to chilblains. This is probably the outcome of endocrine influences, with sympathetic instability and defective calcium assimilation. You will note the resemblance to the type of individual prone to tuberculous infection, and the close analogy between arthritis and tuberculous disease is a feature to which I shall have occasion to return.

We have, then, two aetiological factors-soil and seed ; and there is a third in external influences, such as excessive fatigue-mental or physical-emotional strain, or shock. The combination of cold and damp with unfavourable hygienic conditions depresses resistance to disease generally, though arthritis is by no means a slum disease, and is met with in all classes. Local as well as general influences play a part, and in the less severe types it is noticeable that the joints. first affected are those which are most subjected to strain or which have been the seat of injury. The small proportion of so-called primary cases are almost entirely confined to the female sex, and in these, although no focus of infection can be detected in the light of our present knowledge, it seems not unlikely that here also there is a microbic factor which has by some means obtained access to the system.

A French authority ${ }^{1}$ has recently criticized severely the doctrine of focal infection and the operative treatment of foci. He admits that cure sometimes appears to follow the eradication of septic foci, though in other cases no such happy result ensues; while there are also instances of the arthritis being cured or, it may be, dying out in spite of the continued existence of such foci. He furthermore denies that there is any proof that the streptococci, commonly regarded as the cause, are anything more than common saprophytes; and, while admitting that a focus of infection may serve as a-portal of entry for whatever germ may be responsible for the arthritis, he asserts that we are completely ignorant of its nature. He agrees that

*Address to the Halifax Division of the British Medical Association, November 8th, 1933 . it is sound practice to deal with infective foci in order to put the system into a better state of defence against the infection, whatever it is, and that the extraction of septic teeth, etc., is, for this reason, equally indicated in a case of gonococcal arthritis. While these views will not be accepted in their entirety by workers of experience in this country, they point an important moral-namely, that the treatment of septic foci should be carried out with the aim of improving the general health and never in such a way as to weaken the patient or reduce the powers of resistance through the shock of extensive surgical proceciures. In the primary form, attention to the general health and mode of life is of equal importance, together with treatment of endocrine deficiencies so far as may be possible ; in other respects the principles of treatment for both forms will proceed along the same lines, for there is a strong probability that as our knowledge increases the distinction between the two forms will cease to be made.

\section{Role of the Streptococci in ARthritis}

Opinions vary widely as to the form of streptococcus which is the probable cause of arthritis, and as to whether other microbes may not be equally incriminated. If we admit that the case against the streptococci-haemolytic, anhaemolytic, or viridans, to name the three types most commonly met with in septic foci associated with rheumatic disorders-may not be completely proved, it will still be advisable to look upon them with grave suspicion, and, therefore, to consider the evidence. We are at once faced with the doubts which are arising as to the differences between these three types: Besredka ${ }^{2}$ states definitely that it has been established that a streptococcal infection can take on different clinical forms according to the individual resistance of the subject and according to the ultimate structure of the inoculated region, quite independently of the nature of the streptococcus or of its original source. He holds that the streptococci met with in scarlet fever, for example, may belong to several varieties; and, on the other hand, the same variety of streptococcus may be met with in ciseases clinically different. $\mathrm{He}$ further asserts that demonstrating by means of the microscope the presence of streptococci in the course of an infection is not enough to enable us to conclude that it is a streptococcal illness, a truism which seems sometimes to be forgotten.

Recent researches indicate that there are many culturally different strains of streptococci which may be concerned in the aetiology of rheumatic conditions, that laboratory and cultural distinctions are of less importance than has hitherto been supposed, and that some character common to them all is the important factor in producing tissue reactions of rheumatic type. Some workers believe that it is possible, by suitable cultural methods, to change a haemolytic streptococcus into the viridans type, and thence into the anhaemolytic form. There is also evidence that some, at least, of the diphtheroids which are so frequently met with in cultures from the tonsil may be streptococcal variations. If these changes should be proved our views of the relation of streptococci to certain pathological processes must undergo profound modification. It seems to me also not unlikely that a strepiococcus may be modified in its characters and effects, not only by the tissues in which it grows, but also by other micro-organisms which are present in the same focus. It is very usual to find the Streptococcus viridans associated 
with the Staphylococcus aureus as well as with many other microbes. If we accept the view, as I think we must, that rheumatoid arthritis is commonly associated with the presence of a septic focus and that such focus always contains micrococci of one type or another, the question arises: Do these microbes set up the arthritis, and, if so, how?

Rosenow $^{3}$ believes that crtair. strains of streptococci have a special affinity for joints or connective tissues, and that such affinity may be developed by repeated passages in vivo in rabbits' joints or by other methods ; these strains have been termed " arthrophile." His views are not universally accepted, but cannot lightly be set aside. Of more serious moment are the arguments which seem to be gaining ground that the arthritis which some workers claim to have produced in rabbits by the injection of streptococci does not possess the character of a true rheumatic granulomatosis, but is an ordinary septic arthritis. Magrassi, ${ }^{4}$ who holds this view, claims to have brought about true rheumatic lesions by injecting haemolytic streptococci from rheumatic fever into a joint; a focus is thus formed, and sensitizes certain tissues by means of substances which enter the circulation from it. After an interval of time these substances act as provoking material, and set up local inflammatory reactions or lesions of true rheumatic type. He notes that in such states the intravenous injection of streptococcal antigen will provoke or increase such effects. Although his work has been concerned with rheumatic fever, it seems to have a definite bearing on arthritis. The silent period which has been shown by Glover and others to elapse between an attack of tonsillitis-formation of a focusand the development of an attack of acute rheumatism seems to find an explanation in Magrassi's work. There is considerable evidence in support of the view that the joint and other lesions of rheumatic fever, and also of acute infective or rheumatoid arthritis, are due not to the actual presence of the infecting micro-organism itself in the affected tissues, but to allergic effects. The classical example of allergy in joints is one which many of you will have seen-namely, the arthritis which sometimes follows the injection of horse serum in the form of antitoxin. These allergic reactions may result in animals sensitized to them from the injection of suspensions of streptococci killed by heat, and are analogous to the tissue reactions of rheumatism; but they occur in experimental investigation only in places where an allergic " phlogosis" has been provoked by the introduction of antigen. Swift, Derrick, and Hitchcock, ${ }^{5}$ prominent American workers on this subject, say:

"There is much clinical evidence to support the contention that focal infection has an important bearing on the evolution of this [rheumatic] disease, but up to the present the importance of the focus was thought to rest in its role as a nidus from which the virus was disseminated throughout the body. Our conception of the focus, on the other hand, is of an area where the allergizing substance is produced and whence it is spread to sensitize the various tissues. This conception does not deny that virus may also gain entrance to the blood stream from the focus, but stresses the allergizing effect."

They also observe that "inoculation with non-haemolytic streptococci produces a hyperergic condition and increased susceptibility, not only to the same strain, but also to more distantly related strains."

From these and many other observations on similar Jines has developed the theory that a septic focus continually passes into the blood substances which act upon certain tissues for which they have a predilection in such a way that these tissues become sensitized. Sooner or later a heavy dose is absorbed, or the endocrine defences break down, or the local or general resistance is reduced by one of the many causes already referred to, and inflammation results---an arthritis or fibrositis. This appears to explain adequately the joint lesions of acute rheumatism or the connective tissue reactions of fibrositis, but whether it is sufficient to explain. the persistent and progressive lesions of rheumatoid arthritis without the actual presence of the infecting micro-organisms in the joint tissues is not so evident. In this connexion it is necessary to mention the recent work of Cecil, Nicholls, and Stainsby, ${ }^{6}$ who, by a special technique, have succeeded in growing a streptococcus from the blood. Their work has yet to be confirmed by other observers, but the fact that the organism requires fifteen days to grow suggests that its virulence is low, and strengthens the idea that presensitization of the tissues is essential if it is to exert any pathogenic action.

\section{Rhumatisme TuberculeuX}

Some Continental authorities, especially in France, believe that there is a type of rheumatoid arthritis which is tuberculous in origin. It is claimed that filter-passing forms of the bacillus of Koch have been discovered in these cases. Where a polyarthritis occurs in conjunction with other lesions more generally recognized as tuberculous it is difficult to deny that the joint lesions may also be of that nature. It is claimed, however, that cases of acute polyarthritis resembling acute rheumatism but resistant to salicylates and free from cardiac complications, tending to persistent hydrarthrosis or passing into a chronic polyarthritis, are often tuberculous. Cases of this type in this country are not differentiated clinically from ordinary rheumatoid arthritis, though a few English physicians speak of the Poncet type, after the French physician who first advanced this view. It cannot be denied, however, that the constitutional type and the symptoms, apart from the actual arthritis, are in many cases similar to those of tuberculosis, and that the same general principles of treatment are often indicated. I have examined many cases of this nature without finding evidence of tuberculosis elsewhere, and the von Pirquet and Mantoux tests have proved negative; nor has it fallen to my lot to see any of the cases develop frank tuberculosis. Some of them, however, have given positive reactions to the intradermal vaccine of Ponndorf, without any sign of awakening tuberculous foci elsewhere in the body. Ankylosing spondylitis has also been claimed to be tuberculous, but in this condition also, although its victims are commonly of the tuberculous type, I have not yet been able to satisfy myself that the special lesions were tuberculous; in fact, they are in sharp contrast to the typical appearance and course of tuberculous caries of the spine.

Whatever may be the ultimate outcome of all these theories, one thing at least seems clear-namely, that acute rheumatoid arthritis is usually associated with the presence of septic foci in the body, and presumably is caused by one or more of the micro-organisms therein. Such foci must therefore be the first object of treatment. We must next consider how we may combat the invasion of the system and the joints especially, remembering that in the more acute forms of arthritis there is ample evidence of general constitutional illness, of which the joint lesions are the most obvious.

\section{VACCINE. Therapy}

The question of vaccine treatment may first be con. sidered. It has had a great vogue, but now the pendulum is swinging in the other direction. The success of vaccines in prophylaxis in many diseases led to high hopes that they would be equally successful as a method of treatment, and they have been employed more extensively in chronic rheumatic disease than in any other condition. Faced with a disease which so often pursues 
a steady downhill course the doctor is naturally glad to try any remedy which offers a promise of helping in the fight, especially if it appears to have a scientific basis. Unfortunately the purveying of vaccines has, to a great extent, become commercialized, and the art of advertising has done something to extend their use. From my own observation I should say that there have been more cripples than cures following the injudicious, uninstructed, and often haphazard aciministration of a remedy which with care and skill has sometimes proved of great value in my own experience and in that of many others.

In the leading textbooks on the subject there is a marked lack of enthusiasm. Freund, ${ }^{7}$ a prominent Vienna authority, in his Gelenkerkrankungen, states definitely that he has found no marked differences between the results from vaccine treatment and from non-specific methods. Thompson and Gordon ${ }^{8}$ say that the most that can be expected from vaccines is that they should act as adjuvants to other forms of treatment. They point out that they sometimes appear to be remarkably effective and at other times, in cases apparently identical, there is no benefit whatever. In some instances there is marked reaction to small doses, and in others a large dose produces no reaction at all, making it difficult to give any guidance in treatment. They regard local reaction in the joints as the best guide, and advocate the smallest dose that induces such a reaction. Pemberton ${ }^{9}$ quotes Kolmer, an American authority on immunology, and agrees with his opinion that vaccines made from septic foci, joints, etc., have not given encouraging results. $\mathrm{He}$ also advises that, where there is local reaction in the joints, the dosage should be small and continued over a long period of time. He holds the view that the effects may be non-specific in great measure. Congdon, ${ }^{10}$ in a close statistical analysis of 330 cases of secondary rheumatoid arthritis treated with vaccines in the Devonshire Hospital, Buxton, found a difference of 13 per cent. in favour of cases treated with vaccines. She also showed that stock vaccines gave as good results as autogenous ones: this is opposed to the view of specific therapy. Coste, ${ }^{11}$ after denying that there is any conclusive evidence that the strains of streptococci commonly incriminated have any aetiological relation to arthritis, states as his opinion that all the so-called specific vaccines for arthritis, with the possible exception of gonococcal vaccine and tuberculin, act only by setting up protein shock, to which arthritics are very susceptible. $\mathrm{He}$ expresses doubt even as to the specific effect of gonococcal vaccine in the arthritis of this disease, and suggests that its frequently beneficial action may be owing to some non-specific factor, illustrating this by observations on cases of arthritis which were certainly not of this nature, but which were definitely benefited by gonococcus vaccine. Warren Crowe, ${ }^{12}$ an enthusiastic believer in vaccine therapy in arthritis, claims remarkably good results. $\mathrm{He}$ is of the opinion that a micrococcus closely allied to the common skin staphylococcus is a factor in the procuction of rheumatoid arthritis, and employs a vaccine of this organism as well as a mixed vaccine from a great number of strains ${ }^{\circ}$ of streptococci. $\mathrm{He}$ advocates very small doses, closely regulated by the reactions produced. Cronin Lowe ${ }^{13}$ and other workers have devised what they term the "pathogen selective" method of vaccine therapy. Inoculation of material from the suspected focus is made into the patient's own blood, the theory being that those organisms which survive and are grown on subsequent cultivation are those to which the blood is lacking in protective substances. Apart entirely from the question of vaccine therapy, this procedure seems likely to throw light on the specific character of the microbes in the focus and possibly also on the nature of the infective agent in arthritis.

\section{Rationale of Vaccine Therapy}

The principle involved in injecting a vaccine is generally held to be the stimulation of the formation of antibodies, with the object of strengthening the natural resistance against a specific infection. It is easy to see the preventive value of such a measure, as in the case of inoculation against enteric fever, but the problem becomes far more difficult when it is proposed to use such a vaccine as a method of treatment during the actual course of any disease, when the system is already invaded by the specific microorganism and its toxins, against which the defensive forces of the body are already fully mobilized. Hence, vaccines are empioyed for curative purposes in comparatively few diseases caused by a specific micro-organism, and even in those their value is not universally admitted, and is, indeed, often denied. On the face of it, it would seem illogical, when there is already an invasion of a living and toxin-producing microbe, to inject into the system a series of further doses of the same microbe, even though dead: the dead bodies contain the endotoxin, and in some forms of vaccine the exotoxins are also present. Yet there is clinical evidence that cure has at times followed this line of treatment, and thus, in the case of arthritis, there are enthusiastic advocates of vaccine therapy and others who condemn it with equal energy. It seems probable that it is not a simple matter of antibody formation. Besredka ${ }^{14}$ says definitely that vaccine therapy does not depend on the elaboration of antibodies. To quote his words:

"There are few pathogenic microbes which are as bad producers of antibodies as the staphylococci. However, the clinicians affirm-with a unanimity to which they are but little accustomed-that they know no recoveries more striking than those obtained by vaccino-therapeutic treatment."

His sarcasm at the expense of clinicians may with force be applied to the bacteriologists, for they are sadly lacking in unanimity in the advice they give us. In this problem, as in many others, there is urgent need for more effective liaison between the bacteriologist and the clinician. The former is a rare visitor in the wards, and the latter is not always welcomed in the laboratory; yet the practical value of bacteriology must depend upon the close association of clinical observation with laboratory investigation. Only then will it be possible to lay down with any certainty the proper procedure in treatment, and to decide whether specific or non-specific therapy is likely to yield the best results.

In the face of these consicierations it seems to me that we should employ vaccines of specific type only when an infective focus has been identified and eradicated. We can ignore, I think, the possibility that the infecting organism has become an inhabitant of the bowel, for there is sufficient evidence that bacteria do not normally enter the circulation from this source; and the, toxins, if absorbed, are probably greatly modified, if not cestroyed, by passing through the lines of defence. In such a case we can give vaccines with reasonable confidence, since the supply of the infecting material from the focus has been cut off and we are stimulating the defences of the body without the risk of increasing toxic reactions. Autogenous vaccines may here be the most advantageous, but they must be started in small doses, their effects closely watched, and dosage and interval regulated accordingly. If there is any marked reaction in the joints, or any pyrexial effect, doses must be reduced and intervals increased: such phenomena are likely to be caused by the existence of some unsuspected secondary focus, for which search must be made. An important aspect from the practical point of view of the allergic theory is that, the tissues being sensitized to the toxins, a process of cesensitization is indicated. As asthma 
and hay fever, due to pollens or other non-living substances, are treated by injection of minute doses of the specific substance in order to desensitize the tissues, so may arthritis on the same grounds be treated by vaccines. This is the view of those who believe in extremely small doses, which sometimes, curiously enough, are attended by reactions as definite as those from doses, it may be, a hundred times as great.

\section{Protein Shock}

How far are vaccine effects the result of protein shock is the problem which must next be faced. That arthritics have a he:ghtened susceptib:lity to this has already been mentioned, and the view held by some is that any benefit following vaccines is of this nature. Protein shock induced by non-specific vaccines such as T.A.B., or by the injection of milk, casein, and other special nonbacterial substances such as bee stings, has been tried extensively: in this category must also be placed autohaemotherapy. Such procedures often give good results, but perhaps oftener fail, or relapses occur; as they are apt to be attended by shock, sometimes severe and always attended by risk, the more drastic of these methods are falling into disuse. The employment of stock vaccines as a method of protein shock therapy is less open to objection, and also has the advantage that, if the specific m:crobe is present, it may assist by more direct action. On this principle stock vaccine may be used where a focus is still thought to exist, but with the same precautions as have already been mentioned for autogenous ones. If favourable results are likely to accrue there will soon be signs of this, and if improvement is not quickly seen it is doubtful policy to persevere with the vaccine.

\section{INTRADERMAL VACCINATION}

While vaccines are generally administered by the subcutaneous or intramuscular route, there is another method -namely, intradermal inoculation-which is of proved value in some other bacterial infections, and which is believed to act by stimulating the formation of protective substances from the fixed cells of the deeper layers of the dermis. This has been tried in arthritis, but has not so far been strikingly successful ; it is, however, safer, since absorption is slower, and it is an important feature in a method which is widely employed in France and for which much is claimed, not solely on account of the way of administration, but also because of the vaccines usedthe Ponndorf vaccines. These vaccines are of three types: A, which is derived from bovine tubercle with some proportion of an autolysate of Koch's bacillus; B, which has $\mathbf{A}$ as a basis, with the addition of autolysates of staphylococci, streptococci, pneumococci, and the bacillus of Pfeiffer ; and G, which contains, in addition to $B$ as a base, an autolysate of the gonococcus. All are administered by scarifying the skin and rubbing the vaccine into the scarified area, much as in ordinary vaccination. In rheumatoid arthritis $B$ is indicated, and may give rise to three types of reaction: (1) local, which may vary from slight. redness up to a free serous exudation and formation of a scab; (2) general, which may be absent or severe, with a rise of temperature lasting for a few days; and (3) focal, an increase in the joint syniptoms which, when it occurs, is of good augury.

While some French writers are enthusiastic over this treatment it has not had the same success in the hands of other workers. Personally, in a not very extensive trial, I have had some positive results and some negative ; I. propose to continue its use in suitable cases. (There is a similar preparation, milder in its action-the vaccine of Paul.) It is wise to examine carefully for any tuberculous lesions, active or quiescent, which are generally. held to be a contraindication to the method. It is interesting to note that an English physician, Copeman ${ }^{17}$ adds to the vaccine he employs in some cases a small proportion of tuberculin as a universal non-specific antigen.

\section{CHRYSOTHERAPY}

Chrysotherapy as a way of treating arthritis has recently come into fashion, and is being widely resorted to on the Continent, though, as yet, but little in this country. Coste $^{15}$ asserts that it is the most important add:tion which has been made for a long time to the therapy of chronic rheumatic diseases. We owe much of the work on the subject to Forestier, ${ }^{16}$ who, observing the similarity, between tuberculosis and arthritis, conceived the idea of treating cases of chronic polyarthritis with the salts of gold, which were being therapeutically applied in the former disease. His first publication was an account of forty-four cases, with seventeen very good results, sixteen good results, ten moderate, and only one negative.

Several compounds have been tried, but chiefly allochrysine (an organic compound of gold and sulphur, with sodium) in France, and solganal B (a compound of gold, sulphur, and glucose) in Germany. I have used allochrysine in a certain number of cases with some promising results, but the number is not yet sufficient nor have they been under observation long enough for me to quote. my own experience. The salt is given by the intramuscular route; $\mathbf{5}$ centigrams are given at weekly intervals and the doses are increased cautiously; the treatment is not notably painful. After about twelve weeks, or when a total of 1.5 to 2.5 grams have been given, an interval of six weeks must be allowed for elimination, as the salts of gold are cumulative in the system. Improvement sometimes does not show itself during the first. course, and a second or third may be required before a positive opinion can be formed. The first sign is improvement in general health, followed by diminution of articular swellings, but pain on movement may be diminished much earlier than this, as tested by the amount of aspirin or other analgesics required. In favourable cases the anaemia lessens and the sedimentation rate tends to alter in the direction of normal-an important test.

The principal effects which may result from overdosage or indicate intolerance are: (1) a rise of temperature of half a degree or more, rarely of two or three degrees (which occurred in one of my cases, but was followed by a marked improvement)-this pyrexia is usually confined to the first dose, and if it continues it indicates intolerance; (2) skin eruptions of scarlatiniform or morbilliform type, fleeting in character, and with some itching-this appears to be an anaphylactic reaction, and does not contraindicate treatment. Later symptoms may be a metallic taste, and if this is neglected stomatitis may follow ; the treatment must be temporarily abandoned, or this may become severe. Skin eruptions of a more severe type, and glandular enlargement, may arise. In a few cases albuminuria has been noticed, but almost all these complications followed a dosage much greater than I have mentioned. They indicate, however, the desirability of proper dental toilet and of testing the renal functions beforehand.

Of solganal B I have no personal experience: it is said to be less toxic and of higher therapeutic effect. It has been given by the mouth, and good results are claimed for this method. Skin eruptions may be met with, and one notable symptom as the dose approaches a maximum is fatigue, more or less pronounced, and accompanied by loss of appetite. The focal reactions to the first dose may be more marked than with allochrysine. It is well to examine the blood for any sudden variation in the 
number of leucocytes-a fall in polynuclears especially is an indication to reduce the dose or to suspend treatment. The urine must also be watched for signs of renal irritation. It is necessary to stress the fact that this line of treatment is in its infancy, and needs much careful observation before exact procedures can be laid down.

Recognizing the possible ill effects, one can say that they are no greater than those which accompany vaccine therapy, protein shock, or other methods, and in view of the grave prognosis in untreated cases we are justified in taking some risk, just as the surgeon must regard risks as inseparable from operative measures. The part played by sulphur in these compounds is of interest, as it would appear to increase the activity of the gold acting synergically. This is probably its action when administered independently, as a mineral water, an organic sulphur compound, or otherwise, activating other substances.

\section{Iodine AND CaLcium}

Iodine is a remedy widely employed, and sometimes beneficially. It may be given as the alcoholic solution (thirty grains in an ounce of rectified spirt is a useful prescription), beginning with one drop in milk three times a day and increasing gradually to five or more, with occasional intervals. Or one of the colloidal forms, such as collosol iodine, may be prescribed. The fatty compounds present some advantages, and I have found lipiodol a good one, injected intramuscularly. Compounds of iodine and hexamine are in common use abroad, but I have no experience of them. Iodide of iron with arsenic is a valuable tonic in arthritis.

Calcium is now extensively given because of the evidence of defective calcium metabolism as shown in radiograms by increased transparency of the bones. It is likely that its action is by no means limited to replacing calcium wastage in the bones, and the vogue which the calcium-containing mineral waters have had for centuries for the treatment of rheumatic diseases is suggestive. The intake of calcium in ordinary diet is probably adequate, but the cells appear to have lost their power of assimilating it; it may be that in mineral waters other constituents, such as radon, act by increasing this assimilation in the same way as the action of sulphur already referred to. It is also desirable to include vitamin $\mathrm{D}$ in the form of cod-liver oil or its concentrates to promote assimilation of calcium, or to attain this object by means of exposure to ultra-violet rays.

Many other remedies have been tried in this intractable disease, but time will not permit me to discuss them further. In particular, I regret having to omit all reference to mineral waters and other hydrological methods, and to physical methods of treatment in general, which many authors regard as at least as valuable as vaccines or any other form of internal medication; but you will find these dealt with in the B.M.A. report.

REFERFNCES

'Coste: Traitement du Rhumatisme chronique des Jointures. La Pratique Médicale Illustrée, Paris, 1933.

2 Besredka: Immunity in Infectious Diseases, 1930.

3 Kosenow: Journ. Amer. Med. Assoc., lxv.

- Magrassi: Acta Rheumatologica, 1933, v, 17.

- Swift, Derrick, and Hitchcock: Journ. Amer. Med. Assoc., 1928, xc;Amer. Journ. Med. Sci., clxxxi.

'Cecil, Nicholls, and Stainsby: Journ. Exper. Med., 1929, 1, 617.

"Freund, Ernst: Gelenkerkrankungen, Berlin, 1929.

8 Thompson and Gordon: Chronic Rheumatic Iliseases, 1926.

- Pemberton: Arthritis and Rheumatoid Conditions, 1929.

10 Congdon: Lancet, 1932, i, 178.

11 Coste: Loc. cit.

12 Crowe: Annals of the Pickett-Thomson Research Laboratory, iv, Part 2.

13 Lowe, Cronin: British Medical Journal, July, 1921.

14 Besredka: Loc. cit.

15 Coste: Loc. cit.

16 Forestier: Quoted by Coste, q.v.

"Copeman: Treatment of Rheumatism in General Practice, Arnold, 1933, p. 108.

\section{TREATMENT OF MALARIA IN EUROPEANS BY ATEBRIN}

WITH SPECIAL REFERENCE TO THE RELAPSE RATE BY

\author{
P. D. JOHNSON, M.R.C.S., L.R.C.P. \\ MEDICAL OFFICER, BaTU GAJAH, PERAK, F.M.S.
}

Between June, 1932, and June, 1933, forty-nine European admissions to Batu Gajah Hospital with malaria have been treated by atebrin uncombined with any other specific antimalarial remedy. As all but three of these cases have been followed up for periods ranging from two months to over a year, it has been possible not only to observe the immediate effects of treatment, but also to test the value of this new chemotherapeutic discovery in preventing relapses, for it is in this respect that the greatest claims have been made for atebrin. Of these forty-nine cases, twenty-nine were diagnosed microscopically as benign tertian, seventeen as subtertian, and three as mixed tertian malaria. Atebrin was given in the form of 0.1 gram tablets three times a day between meals, children receiving a proportionately smaller dose. The total adult dose varied between 1.4 and 2.4 grams. Complete rest in bed was maintained for forty-eight hours after the termination of the febrile period, and, apart from a preliminary dose of calomel, a daily saline purgative, and aspirin during the " hot stage," no other drugs were prescribed as a routine.

\section{Immediate Effects of Treatment}

Compared with eighty-three European cases treated between June, 1931, and June, 1932, by quinine, plasmoquine, and alkalis, the treatment by atebrin has proved very satisfactory. A large proportion of the quininetreated subtertian cases were given intramuscular injections on account of vomiting, but in only one instance had this to be resorted to in the atebrin-treated cases. It may be that a particularly mild type of infection was encountered in the 1932-3 series, but the impression remains that quinine by the mouth very frequently excites vomiting. In both series subtertian malaria took rather longer to control than benign tertian, but atebrin seemed superior in this respect to quinine. It was found comparatively pleasant to take, and did not cause any of the neurotropic symptoms so frequently associated with quinine. For this reason it was particularly valuable in children, who tolerated doses rather larger than those usually recommended. One infant, aged 8 months, was given 0.1 gram daily for five days without any ill-effects. Many patients expressed a feeling of well-being during treatment, and those with previous experience of quinine were unanimous in their preference for atebrin.

Malaria complicating pregnancy was treated in three cases by quinine and in two cases by atebrin. On no occasion did quinine or atebrin appear to have any deieterious effect on the pregnant uterus. One patient with an idiosyncrasy to quinine developed urticarial and pseudo-asthmatic symptoms after 5 grains, but was able to take a full course of atebrin without any ill effects. A comparison between the duration of the pyrexial period and the persistence of malaria parasites in the blood after the beginning of treatment is given in Table $I$. The quinine-treated cases received 2 grams of quinine and 0.01 gram of plasmoquine daily.

As far as the immediate treatment is concerned, it would appear from these figures that atebrin is superior to quinine in controlling the temperature and ridding the blood of parasites in all types of malaria, but particularly so in subtertian infections. Its occasional provocative effect on the parasites of subtertian malaria will be discussed later. 